\title{
Correction to: Food conditions, competitive regime, and female social relationships in Japanese macaques: within-population variation on Yakushima
}

\author{
Goro Hanya ${ }^{1}$. Miki Matsubara ${ }^{1}$. Shuhei Hayaishi ${ }^{2} \cdot K$ Koichiro Zamma ${ }^{3}$ Shinichi Yoshihiro ${ }^{4}$ Masahiro M. Kanaoka ${ }^{5}$. \\ Shuji Sugaya ${ }^{6} \cdot$ Mieko Kiyono $^{5} \cdot$ Makiko Nagai $^{7} \cdot$ Yosuke Tsuriya $^{8} \cdot$ Sachiko Hayakawa $^{1} \cdot$ Mariko Suzuki $^{1}$. \\ Takashi Yokota $^{9} \cdot$ Daisuke Kondo $^{10} \cdot$ Yukio Takahata $^{11}$
}

Published online: 26 February 2021

(C) Japan Monkey Centre 2021

\section{Correction to: Primates (2008) 49:116-125 https://doi.org/10.1007/s10329-007-0073-y}

In our published original paper, we found an error in a figure described in the sentence starting on line 14 on the right column of page 118. We correct the sentence as follows:

"The mean density of food trees that comprised at least $1 \%$ of the annual feeding time was 1,493 trees/ha in the coniferous forest (Hanya 2004b), which is much larger than that in the coastal forest (505 trees/ha; Agetsuma 1995; Agetsuma, unpublished data)".

This correction does not affect other parts of this paper, including the conclusion. The authors apologize sincerely for this error.

Publisher's Note Springer Nature remains neutral with regard to jurisdictional claims in published maps and institutional affiliations.

The original article can be found online at https://doi.org/10.1007/ s10329-007-0073-y.

Goro Hanya

hanya.goro.5z@kyoto-u.ac.jp

1 Primate Research Institute, Kyoto University, Inuyama, Aichi 484-8506, Japan

2 University of Ryukyus, Okinawa 901-2214, Japan

3 Japan Monkey Centre, Inuyama 484-8501, Japan

4 Ryukoku University, Kyoto 612-8577, Japan

5 Graduate School of Science, Kyoto University, Kyoto 606-8502, Japan

6 Higashino, Kasugai 486-0817, Japan

7 Yokohama National University, Yokohama 240-8501, Japan

8 Nihon University, Fujisawa 252-8510, Japan

9 Graduate School of Informatics, Kyoto University, Kyoto 606-8501, Japan

10 Gifu University, Gifu 501-1193, Japan

11 Kwansei-Gakuin University, Sanda 669-1337, Japan 